\title{
PSO-ANN in preventing traffic collisions: a comparative study
}

\author{
Md. Ashikuzzaman, Wasim Akram, Md. Mydul Islam Anik, Mahamudul Hasan, Md. Sawkat Ali, \\ Taskeed Jabid \\ Department of Computer Science and Engineering, East West University, Dhaka, Bangladesh
}

\begin{abstract}
Article Info
Article history:

Received Sep 13, 2020

Revised Oct 19, 2021

Accepted Oct 26, 2021

\section{Keywords:}

Hybrid artificial neural network

Machine learning model

Particle swarm optimization

Prediction accuracy

Traffic collision

ABSTRACT

Traffic accident is a global threat which causes health and economic casualties all around the world. Due to the expansion of transportation systems, congestion can lead to spike road accident. Every year thousands of people have died due to traffic accidents. Various technologies have been adopted by modern cities to minimize traffic accidents. Therefore, to ensure people's safety, the concept of the smart city has been introduced. In a smart city, factors like road, light, and weather conditions are important to consider to predict traffic mishap. Several machine learning models have been implemented in the existing literature to determine and predict traffic collision. But the accuracy is not enough and there exist a lot of challenges in determining the accident. In this paper, an approach of particle swarm optimization with artificial neural network (PSO-ANN) has been proposed to determine traffic collision using the dataset of the transport department of United Kingdom. The performance of PSO-ANN outperforms the existing machine learning model. PSO-ANN model can be adopted in the transportation system to counter traffic accident issues. Random Forest, Naïve Bayes, Nearest Centroid, K-Nearest Neighbor classification have been used to compare with the proposed PSO-ANN model.
\end{abstract}

This is an open access article under the CC BY-SA license.

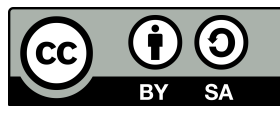

\section{Corresponding Author:}

Mahamudul Hasan

Department of Computer Science and Engineering

East West University

Dhaka, Bangladesh

Email: munna09bd@gmail.com

\section{INTRODUCTION}

In this modern world, the traffic accident has already become a matter of concern. People are losing their life due to this disastrous incident. Life becoming difficult day by day. An accident not only causes a huge damage to the family but also can affect the economy of a country. This incident has become a barrier to the development of a country and eventually causes great damage globally. Economy of the Americas and Europe largely depend on on-road transportation. Even a promising country like Malaysia faces 24 deaths among every 100,000 people which is a very high number [1]. According to world statistics, the total number of accident fatalities in 2016 was 1,350,000 worldwide. In several regions like Africa, Europe, America, South-east Asia, Western Pacific, the fatalities number was 246,719, 85,629, 153,789, 316,080, and 328,591 respectively [2]. In the UK the fatality number was 2,019 in 2016. Traffic accidents act as resistance to normal day-to-day life [3].

We witness the incident of road accidents regularly. Some main reasons are driving carelessly, traffic rule violations, and most importantly the increased number of vehicles [4]. Chong et al. tried to find out factors 
that are significant to determine the severity of injury of the injured in traffic accidents [5].

Machine learning can be used to predict traffic mishap that will help to save lives. The damage and the injury can be rescued and even lives can be saved with the prompt arrival of assistance through a smart system developed using these models. Several approaches of machine learning models such as decision tree, support vector machine (SVM), regression model, random forest, and naïve bayes. are used to get good results in traffic collision prediction, but most of them are not well optimized for big datasets. For example, SVM is a non-parametric classifier machine learning model. Non-parametric classifiers are slower to train as they often have to train more parameters. They required more training data to estimate the mapping function. Again, there is also the risk of overfitting while training data with a non-parametric classifier. To overcome this overfitting problem, artificial neural network (ANN) is used which also performs better for large datasets and complex computing. It is a parametric classifier. However, ANN models are more prone to stuck in local minima. Hybridizing ANN with particle swarm optimization (PSO) will reduce this problem as the PSO algorithm finds global optima efficiently and quickly. The hybrid algorithm of PSO-ANN is shown on Algorithm 1. Based on the outcome of previous researches, the PSO-ANN method has been proposed in this study to detect the collision of the traffic [6].

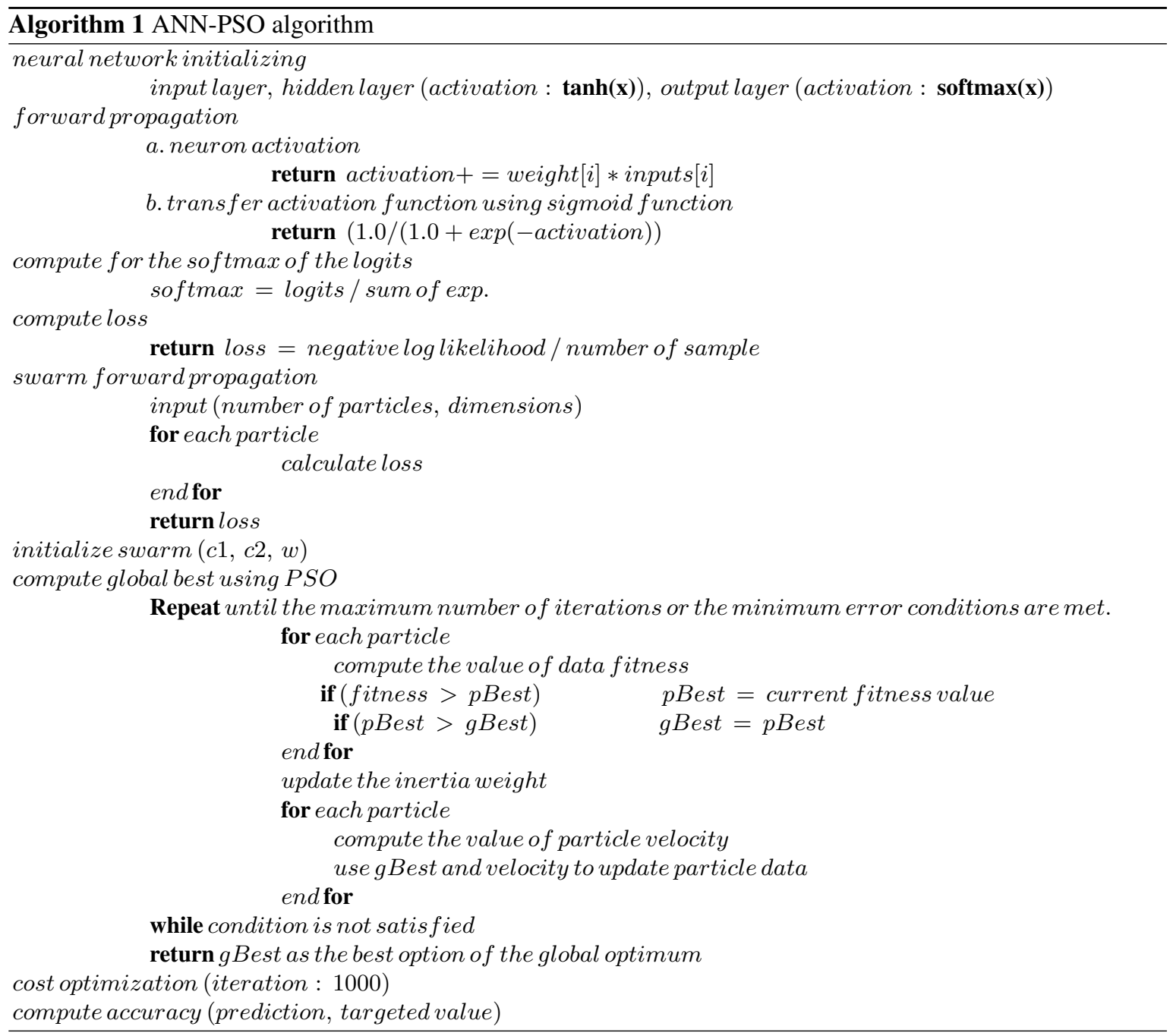

\section{RELATED WORK}

There has been a lot of research into predicting the traffic collisions. Recurrent neural network (RNN) was employed in traffic collision severity prediction in a study conducted by Sameen and Pradhan, based on 1130 accident data that happened on the north-south expressway (NSE) in Malaysia between 2009 and 2015. 
The dataset was split into two parts: 80 percent for training and 20 percent for validating and the RNN model was used to calculate the result for 100 iterations. The accuracy of the RNN model was 71.77 percent. On the other hand, multilayer perceptron (MLP) and bayesian logistic regression (BLR) models achieved 65.48 percent and 58.30 percent accuracy respectively. As a result, the RNN model, which outperformed the MLP and BLR models, was chosen [7].

Alkheder and Tammneh proposed two models such as the ordered probit model and ANN to examine the severity of traffic accidents [8]. The Probit model is a form of regression model that examines the relationship between categorical and numerical variables [9]. In traffic accident data from the Emirate of Abu Dhabi, the ANN and the ordered probit model were employed (2008-2013). At the time of the accident, 48 distinct attributes were collected from 5973 separate instances. The ordered probit model had a lower accuracy of 59.5 percent than the ANN model, and the ANN model had a higher accuracy of 74.6 percent for traffic collision prediction [8].

Al-Raddaideh and Daoud investigated the prediction of traffic accidents using three categorization approaches: random forest (RF), artificial neural networks (ANN), and support vector machines (SVM). Cleaning, normalization, feature selection, and transformation were performed on the dataset. The dataset was divided into two parts: $66 \%$ for training and $34 \%$ for testing, with 10-fold Cross-Validation applied. The RF model had an accuracy of 80.6 percent, while the ANN and SVM models had 61.4 percent and $54.8 \%$ accuracy, respectively. As a result, RF will be an apparent choice for anticipating the severity of a traffic accident due to its more trustworthy decision-making process [10]. In a study of predicting crash injury severity, fuzzy c-means clustering-based support vector machine (SVM-FCM) shows better result than fuzzy c-means clustering-based feed-forward networks (FNN-FCM). The crash database of the United Kingdom from 2011 to 2016 was used in this study [11].

When it comes to predicting collision, Bayesian networks are more useful. Zong et al. discovered that Bayesian networks outperformed the regression model in predicting the severity of traffic accidents. The dataset in this case contained police-reported traffic accident records from the Chinese province of Jilin in 2010 [12]. According to Zheng et al. and colleagues, the TASP-CNN model performed better for predicting the severity of traffic accidents. The weights of each feature of the traffic collision were calculated, and the FM2GI technique was used to convert the feature matrix to a gray image. The method entails converting a single feature relationship from the accident dataset into gray photos, then combining it as an input variable to test or train the model in parallel [13].

To forecast traffic flow, the particle swarm optimization (PSO) was combined with an artificial neural network (ANN) [14]. PSO is popular because of its speed and ability to search in high-dimensional problem spaces [15]. According to B. Bashumathi and S. Moorthi's research, ANN-PSO is useful for harmonic estimation in active filters, and it converges to a about 50-60\% lower error value than the Adaline approach [16]. PSO-ANN has an accuracy of 82.42 percent for detecting skin illness, according to Chakraborty's et al. research. More than 25000 photos of various diseases are included in the dataset [17]. In a hybrid PSO-ANN study, Darmawan and colleagues demonstrated that a person's sex can be inferred using the bone length of the left hand. However, when looking at the age groups of 16-19 and 7-9, these two age groups exhibited superior outcomes than the other age groups [18], with an average of $80 \%$ above correctness.

\section{METHODOLOGY}

The dataset is encoded into numerical representation from categorical data using "One Hot" encoding technique. After encoding, training and testing data is split into 70-30\% ratio. The input layer, hidden layer, and output layer sizes were set to 12, 5 and 3 respectively to initialize the neural network. The "tanh" function was utilized as an activation function in the hidden layer, and "softmax" was employed as an activation function in the output layer. The hidden layer accepts the input data and processes it using an activation function in forward propagation. The activation function is responsible for making a neural network non-linear [19]. The tangent, hyperbolic, sigmoid, and linear functions are commonly chosen activation functions [20].

The softmax function is applied to the logits layer, which holds raw predictions, in order to bring the result closer to one or zero. The softmax function [21] is used to calculate cross-entropy loss, which is based on the corresponding probability of the network output of the proper category. After that, we built a function that will propagate over the entire swarm. Swarm is initialized, and then PSO is used to compute the global best solution. 
The position of particles in PSO has been upgraded to provide the current position, personal best position, and swarm's global best position. Particles calculate their distance from each other and migrate toward the eventual ideal position of the swarm in each iteration [22]. The process was repeated until the 1000th iteration. Finally, the prediction accuracy has been calculated using the test set. In our approach of implementing particle swarm optimization (PSO) with artificial neural network (ANN), the (1), (2), and (3) are used:

$$
v_{m n}^{t+1}=v_{m n}^{t}+c_{1} r_{l n}^{t}\left[P_{\text {best }, m}^{t}-x_{m n}^{t}\right]+c_{2} r_{2 n}^{t}\left[G_{\text {best }}-x_{m n}^{t}\right]
$$

$v^{t} m n$ is the velocity vector of particle $m$ in dimension $n$ at time $t$, according to (1), at time $t, x_{m n}^{t}$ is the velocity vector of particle $m$ in dimension $n$. And $P_{\text {best } m}^{t}(2)$ is particle $m$ 's personal best location in dimension $n$ as determined from startup to time $t$. And from initialization through time $t, G_{\text {best }}(3)$ is the global best location of particle $m$ in dimension $n, r^{t} 1 n$ and $r^{t} 2 n$ are random integers from uniform distribution $U(0,1)$ at time $t, c 1$ and $c_{2}$ are positive acceleration constants that are employed to level the contribution of the cognitive and social components, respectively.

$$
\begin{gathered}
P_{\text {best }, m}^{t+1}= \begin{cases}P_{\text {best }, m}^{t} & \text { if } f\left(x_{m}^{t+1}\right)>P_{\text {best }, m}^{t} \\
x_{m}^{t+1} & \text { if } f\left(x_{m}^{t+1}\right) \leq P_{\text {best }, m}^{t}\end{cases} \\
G_{\text {best }}=\min \left\{P_{\text {best }, m}^{t}\right\}, \text { where } m \in[1, \ldots, x] \text { and } x>1
\end{gathered}
$$

\section{OVERVIEW OF DATASET}

Road Accident data has been utilized in this research which has been published by the Transport Department of the United Kingdom [23]. This dataset is related to environmental factors containing 2203 traffic accident records with 15 features. Reference number and accident date features are not taken into consideration in the pre-processing step. So, in total 13 features including the class labels are being utilized for this research. The 13 features are "Easting ( Represents integer type values situated horizontally)", "Northing (Represents integer type values situated vertically)", "Number of Vehicles", "The time of the accident occurred (Values are integer types)", "1st Road Class", "Road Surface (Wet, Dry, Snow, Frost)", "Lighting Conditions (Daylight: Street lights present, Darkness: Street lights present and lit, No street lighting, and Street lighting unknown)", "Weather Conditions", "Type of Vehicles", "Casualty Class (Pedestrian, Driver or rider, Vehicle or pillion passenger)", "Casualty Severity (Serious, Slight, Fatal)", "Sex of Casualty (Female, Male)" and "Age of Casualty (Unique Ages sample)". Slight, fatal and serious are the three classes found in Figure 1 (a). The pie chart shows that percentages of the class label. According to Figure 1 (b), age of under 80 people had fatal injuries. The severity level slightly occurred to most of all aged people. From the analysis, it is found that the group of people aging above 60 has a higher risk to be seriously injured in a traffic accident.

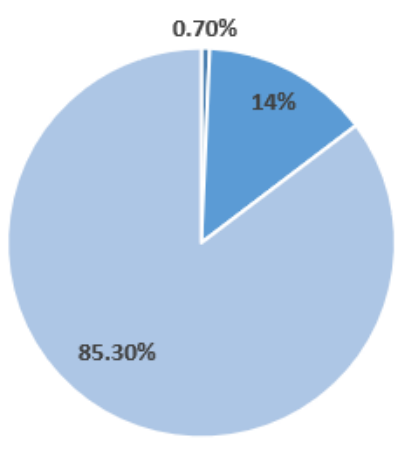

(a)

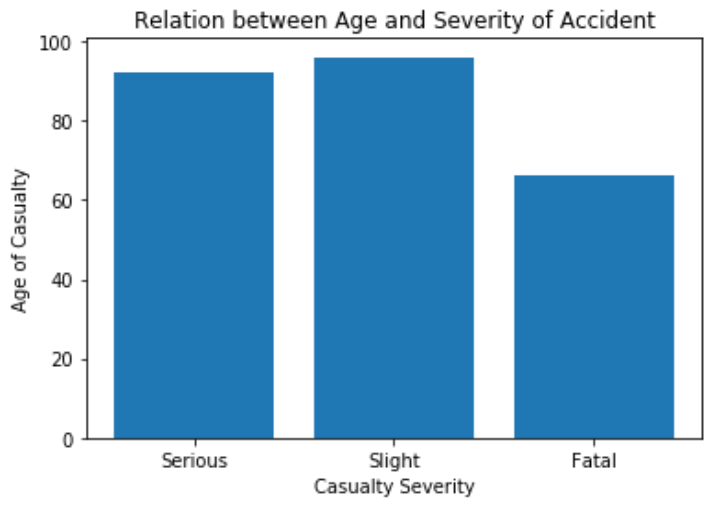

(b)

Figure 1. Severity analysis of dataset using pie chart and bar chart: (a) class label is shown in the pie chart with a percentage value and (b) bar chart of the relation between age and severity of accident 


\section{RESULT ANALYSIS AND DISCUSSION}

In this section, the hybridized neural network and other models are being analyzed based on accuracy, precision, recall, and f1-score [24], [25]. Table 1 shows an accuracies comparison between PSO-ANN and other machine learning models. The accuracy of PSO-ANN and Random forest is pretty close for this dataset.

Table 1. Accuracy table of different models

\begin{tabular}{lc}
\hline Name of Algorithms & Accuracy \\
\hline PSO-ANN & $85.29 \%$ \\
Random Forest & $85.04 \%$ \\
Nä̈ve Bayes & $84 \%$ \\
K Nearest Neighbor & $75.18 \%$ \\
Nearest Centroid & $55 \%$ \\
\hline
\end{tabular}

In Figure 2 (a) the measurement of precision, recall, and f1-score of all the machine learning models are shown with the bar chart. With Figure 2 (b) the accuracy of nearest centroid, K-Nearest Neighbor, Naive Bayes, Random Forest, and our proposed approach PSO-ANN have been shown. Both figures illustrate the superiority of PSO-ANN over other machine learning models. In Figure 3, the relationship between cost and iteration is illustrated. The cost is drastically reduced with each iteration. As iteration increases cost tensed to be close to zero.

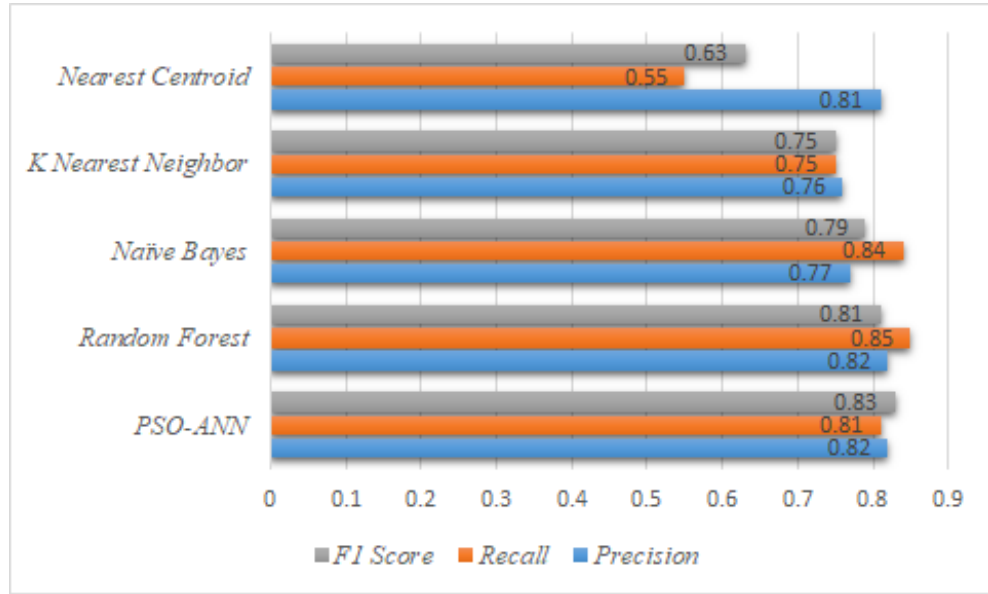

(a)

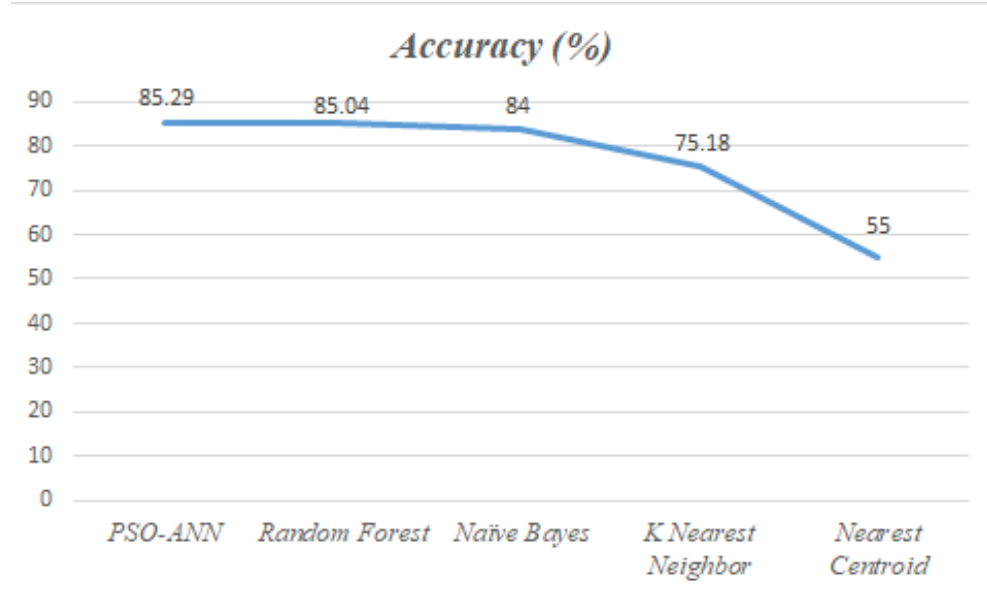

(b)

Figure 2. Superiority of PSO-ANN over other machine learning models: (a) bar chart and (b) line chart of analysis of accuracy, precision, recall and f1-score 


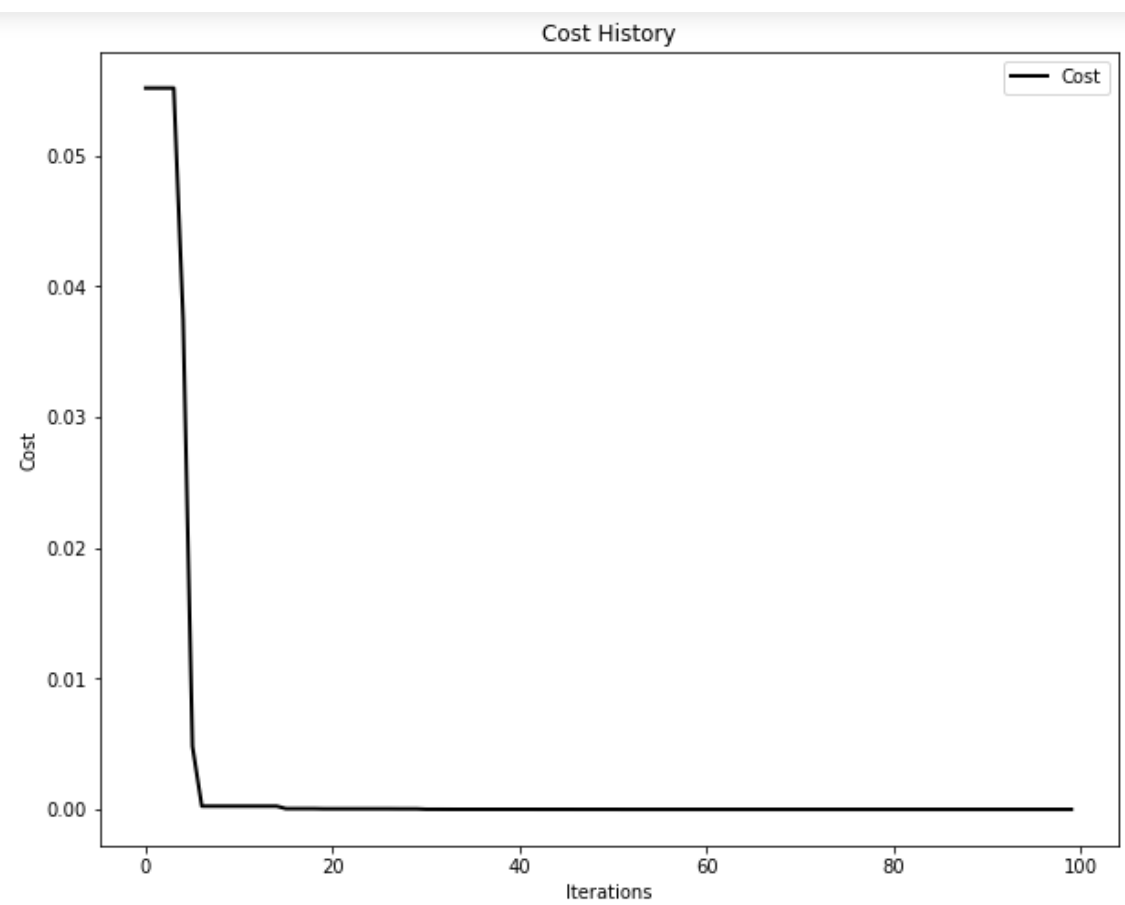

Figure 3. Iteration vs cost

The best accuracy is obtained by the PSO-ANN model which is $85.29 \%$. Whereas, the lowest accuracy result is achieved by the nearest centroid which was 55\%. So, PSO-ANN can be considered as more reliable and accurate to give collision prediction of traffic. F1-score is more convenient than accuracy in terms of uneven class distribution. Figure 2 shows that the highest $\mathrm{f} 1$-score of 0.83 which is obtained by PSO-ANN. Again, the highest precision score of 0.82 is obtained by PSO-ANN and Random Forest, highest recall of 0.85 is secured by Random Forest whereas recall of 0.81 is achieved by PSO-ANN.

\section{CONCLUSION}

Road accidents should not be a deterrent to a country's progress. This economic problem can be alleviated if the damage, as well as traffic accidents, can be decreased. It is necessary to raise public awareness in order to reduce traffic collisions. However, if the appropriate procedures are performed in a timely manner, the damage can be limited. To solve this difficulty, a wide range of forecasting models have been presented. These models progressed from linear to nonlinear forms, as well as from traditional statistical regression models to today's most popular machine learning models. If we can forecast the severity of an accident, we can take the necessary precautions. The sooner we can address the problem created by the traffic accident, the sooner we can return to normalcy. This will allow people to continue working as usual. As a result, a country's economy will suffer less damage. Many Machine Learning approaches utilized to detect the severity of a traffic collision in this study. The hybrid form of partical swarm optimizer and artificial neural network (PSO-ANN) are compared using naive bayes, random forest, k-nearest neighbor, and nearest centroid (ANN). The PSO-ANN outperforms all of the other models.

\section{REFERENCES}

[1] M. I. Sameen and B. Pradhan, "Assessment of the effects of expressway geometric design features on the frequency of accident crash rates using high-resolution laser scanning data and GIS," Geomatics, Natural Hazards and Risk, vol. 8, no. 2, pp. 733-747, 2017, doi: 10.1080/19475705.2016.1265012.

[2] WHO, "Deaths on the roads: Based on the WHO Global Status Report on Road Safety 2015," Official report. Geneva, Switzerland: World Health Organisation (WHO), Jan 26, 2016. [Online]. Available: https://www.afro.who.int/publications/global-status-report-road-safety-2015 
[3] M. F. Coelho, J. M. Bandeira and M. C. Coelho, "Impact of road traffic incidents on pollutant emissions," 2011 IEEE Forum on Integrated and Sustainable Transportation Systems, 2011, pp. 312-316, doi: 10.1109/FISTS.2011.5973633.

[4] E. Johnson, J. M. Abraham, S. Sulaiman, L. Padma Suresh and S. Deepa Rajan, "Study on Road Accidents Using Data Mining Technology," 2018 Conference on Emerging Devices and Smart Systems (ICEDSS), 2018, pp. 250-252, doi: 10.1109/ICEDSS.2018.8544370.

[5] M. Chong, A. Abraham and M. Paprzycki, "Traffic accident data mining using machine learning paradigms," Fourth International Conference on Intelligent Systems Design and Applications (ISDA'04), Hungary, pp. 415-420, 2004.

[6] M. F. Labib, A. S. Rifat, M. M. Hossain, A. K. Das and F. Nawrine, "Road Accident Analysis and Prediction of Accident Severity by Using Machine Learning in Bangladesh," 2019 7th International Conference on Smart Computing \& Communications (ICSCC), 2019, pp. 1-5, doi: 10.1109/ICSCC.2019.8843640.

[7] M. I. Sameen and B. Pradhan, "Severity prediction of traffic accidents with recurrent neural networks," Applied Sciences, vol. 7, no. 6, 2017, doi: 10.3390/app7060476.

[8] S. Alkheder and M. Taamneh, "Severity Prediction of Traffic Accident Using an Artificial Neural Network," Journal of Forecasting, vol. 36, no. 1, pp. 100-108, 2016, doi: 10.1002/for.2425.

[9] T. F. Dewanto, V. Ratnasari and Purhadi, "Spatial probit regression model: Recursive importance sampling approach," 2018 International Conference on Information and Communications Technology (ICOIACT), 2018, pp. 759-764, doi: 10.1109/ICOIACT.2018.8350785.

[10] Q. A. Al-Radaideh and E. J. Daoud, "Data mining methods for traffic accident severity prediction," International Journal of Neural Networks and Advanced Applications, vol. 5, pp. 1-12, 2018.

[11] K. Assi, S. M. Rahman, U. Mansoor and N. Ratrout, "Predicting crash injury severity with machine learning algorithm synergized with clustering technique: a promising protocol," International journal of environmental research and public health, vol. 17, no. 15, 2020, doi: 10.3390/ijerph17155497.

[12] F. Zong, H. Xu and H. Zhang, "Prediction for traffic accident severity: Comparing the bayesian network and regression models," Mathematical Problems in Engineering, vol. 2013, 2013, doi: 10.1155/2013/475194.

[13] M. Zheng et al., "Traffic accident's severity prediction: A deep-learning approach-based CNN network," IEEE Access, vol. 7, pp. 39897-39910, 2019, doi: 10.1109/ACCESS.2019.2903319.

[14] L. D. Zhang, L. Jia and W. X. Zhu, "Overview of traffic flow hybrid ANN forecasting algorithm study," 2010 International Conference on Computer Application and System Modeling (ICCASM 2010), 2010, pp. V1-615-V1-619, doi: 10.1109/ICCASM.2010.5620414.

[15] T. Hendtlass, "Particle swarm optimisation and high dimensional problem spaces," 2009 IEEE Congress on Evolutionary Computation, 2009, pp. 1988-1994, doi: 10.1109/CEC.2009.4983184.

[16] B. Vasumathi and S. Moorthi, "Implementation of hybrid ANN-PSO algorithm on FPGA for harmonic estimation," Engineering Applications of Artificial Intelligence, vol. 25, no. 3, pp. 476-483, 2012, doi: 10.1016/j.engappai.2011.12.005.

[17] S. Chakraborty et al., "Detection of skin disease using metaheuristic supported artificial neural networks," 2017 8th Annual Industrial Automation and Electromechanical Engineering Conference (IEMECON), 2017, pp. 224-229, doi: 10.1109/IEMECON.2017.8079594.

[18] M. F. Darmawan, S. M. Yusuf, M. A. Rozi and H. Haron, "Hybrid PSO-ANN for sex estimation based on length of left hand bone," 2015 IEEE Student Conference on Research and Development (SCOReD), 2015, pp. 478-483, doi: 10.1109/SCORED.2015.7449382.

[19] R. K. Yadav and Anubhav, "GA and PSO hybrid algorithm for ANN training with application in Medical Diagnosis," 2019 Third International Conference on Intelligent Computing in Data Sciences (ICDS), 2019, pp. 1-5, doi: 10.1109/ICDS47004.2019.8942375.

[20] J. Lee, T. Yoon, S. Kwon, and J. Lee, "Model evaluation for forecasting traffic accident severity in rainy seasons using machine learning algorithms: Seoul city study," Applied Sciences, vol. 10, no.1, 2020, doi: 10.3390/app10010129.

[21] Q. Zhu, Z. He, T. Zhang, and W. Cui, "Improving Classification Performance of Softmax Loss Function Based on Scalable Batch-Normalization,” Applied Sciences, vol. 10, no. 8, 2020, doi: 10.3390/app10082950.

[22] M. H. Mofrad and S. K. Chang, "A Bi-population Particle Swarm Optimizer for Learning Automata based Slow Intelligent System," arXiv preprint arXiv:1804.00768, 2018.

[23] Open Government Licence, "Road traffic accidents," Leeds City Council, July 22, 2020. Accessed: May $20,2021$. Url: https://data.gov.uk/dataset/6efe5505-941f-45bf-b576-4c1e09b579a1/road-traffic-accidents

[24] M. G. Sarowar, F. Qasim, and S. H. Ripon, "Tuberous Sclerosis Complex (TSC) Disease Prediction Using Optimized Convolutional Neural Network," ICCCM 2019: Proceedings of the 2019 7th International Conference on Computer and Communications Management, 2019, pp. 210-215, doi: 10.1145/3348445.3348454.

[25] B. Sahu, S. N. Mohanty, and S. K. Rout, "A hybrid approach for breast cancer classification and diagnosis," EAI Endorsed Transactions on Scalable Information Systems, vol. 19, no. 20, 2019, doi: 10.4108/eai.19-12-2018.156086. 


\section{BIOGRAPHIES OF AUTHORS}

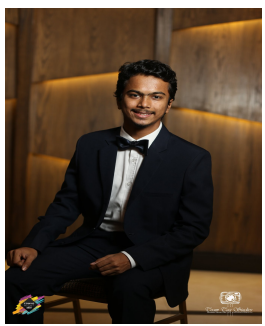

Md. Ashikuzzaman is a computer engineer, a graduate research assistant, and a software developer intern. He earned his Bachelor's degree from East West University with exemplary academic performance and leadership skills. His research interest are in fields of Machine Learning, Deep Learning, Image Processing.

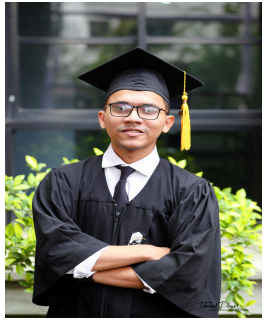

Wasim Akram is a Research Assistant and a former Graduate Teaching Assistant at East West University, Bangladesh. He is currently working as a Software Engineer. He earned his Bachelor Degree in Computer Science and Engineering from East West University in 2020. His research interest are in fields of Machine Learning, Deep Learning, Image Processing.

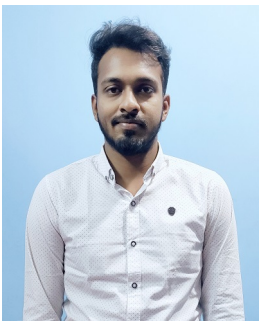

Mydul Islam Anik is a graduate in Computer Science and Engineering. He completed his bachelor's degree in the field of Computer Science and Engineering from East West University, Bangladesh. He is currently working as a Junior Software Engineer in a leading software firm in Bangladesh. His researches include Machine Learning, Data mining, Deep Learning, etc.

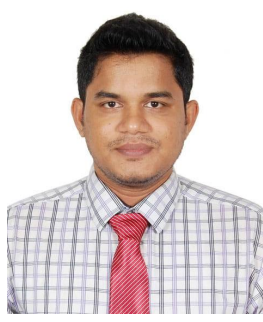

Mahamudul Hasan is a Senior Lecturer at East West University. He obtained Masters and Bachelor Degree in Computer Science and Engineering from University of Dhaka (Bangladesh) in 2015 and 2014 respectively. He has several publication in the field of Recommender System, Computer Vision, Data Science, Machine Learning and so on. His researches are in fields of Recommender System, Machine Learning, Deep Learning, Image Processing.

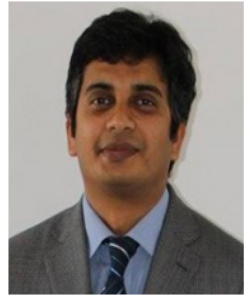

Md. Sawkat Ali received his B.Sc. degree in Electrical and Electronic Engineering (EEE) from the Ahsanullah University of Science and Technology, Bangladesh. He completed M.Eng. (research) degree in Electrical Engineering from the University of New South Wales, Canberra, ACT, Australia, and Ph.D. degree in Electrical Engineering from Central Queensland University, Rockhampton, Australia. He worked as a Post-Doctoral research fellow at Deakin University, Australia. He is currently working as an Assistant Professor with the Department of Computer Science and Engineering, East West University, Dhaka, Bangladesh. His research interests include power electronics, linear, nonlinear control theory and its applications, IoT, microgrids, renewable energy, machine learning.

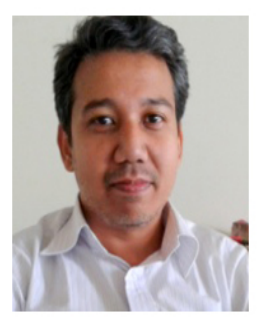

Taskid Jabid is an Associate Professor at East West University. Taskid Jabid received his Ph.D. in Computer Vision and Image Processing, Kyung Hee University, South Korea. He obtained his Bachelor Degree in Computer Science and Engineering from East West University (Bangladesh). His researches are in fields of Machine Learning, Deep Learning, Image Processing, Facial Image Analysis, Texture Analysis, Bioinformatics, Computer, Graphics, Algorithms. 